BMJ

Open

Gastroenterology

\title{
Combination therapy with vedolizumab and etanercept in a patient with pouchitis and spondylarthritis
}

\author{
Johannes Bethge, Silvia Meffert, Mark Ellrichmann, Claudio Conrad, \\ Susanna Nikolaus, Stefan Schreiber
}

To cite: Bethge J, Meffert S, Ellrichmann $\mathrm{M}$, et al. Combination therapy with vedolizumab and etanercept in a patient with pouchitis and spondylarthritis. BMJ Open Gastro 2017;4: e000127. doi:10.1136/ bmjgast-2016-000127

Received 1 November 2016 Revised 15 December 2016 Accepted 20 December 2016
Medical Department I, Gastroenterology, University Medical Center, Schleswig Holstein, Campus Kiel, Kiel, Germany

\section{Correspondence to}

Professor Stefan Schreiber; s.schreiber@mucosa.de and Dr Johannes Bethge; johannes.bethge@uksh.de

\section{ABSTRACT}

Inflammatory bowel disease is frequently associated with spondylarthritis (SpA). It has been discussed that $\alpha 4 / \beta 7$ expressing lymphocytes are involved in the aetiology of SpA. We report a case of a successful combination therapy of vedolizumab (VDZ) and etanercept (ETA) in a patient with ulcerative colitis with pouchitis and SpA. In our case VDZ was effective for pouchitis and ineffective for SpA. The combination with ETA might be a useful treatment strategy to control both diseases and first indications suggest that it is safe. $\alpha 4 / \beta 7$ Expressing lymphocytes are most likely not associated in the aetiology of SpA.

\section{INTRODUCTION}

Inflammatory bowel disease (IBD) is frequently associated with independent inflammatory comorbidities including spondylarthritis (enteropathic seronegative spondylarthritis $(\mathrm{SpA})$ ), rheumatoid arthritis or psoriasis.

SpA is one of the most frequent extraintestinal manifestations (EIM) in patients with IBD. ${ }^{1}$ About a third of patients with IBD suffer from arthropathies and in 5-12\% SpA can be observed. ${ }^{2}$ Vice versa, about $7 \%$ of patients with SpA develop IBD during the course of their disease. ${ }^{3}$

It has been discussed that $\alpha 4 / \beta 7$ expressing lymphocytes, as part of the gut-joint axis in IBD, are involved in the aetiology of SpA. ${ }^{2} 4$ Established treatments of SpA include glucocorticoids, sulfasalazine, azathioprine, methotrexate and TNF-inhibitors.

Pouchitis is the main complication after proctocolectomy with ileal pouch-anal anastomosis (IPAA) in patients with ulcerative colitis (UC). Most patients respond to antibiotic treatment. ${ }^{5}$ About 5-19\% patients develop a chronic relapsing or treatment-refractory disease. For patients who suffer from chronic antibiotic refractory pouchitis some alternative immunosuppressive therapy options are successful (ie, anti-TNF biologics). ${ }^{6}$

\section{Summary box}

What is already known about this subject?

- Tumour necrosis factor (TNF) inhibitors are an established treatment option to control spondylarthritis.

- Gut-inflammation in patients with inflammatory bowel disease (IBD) shows good response to vedolizumab, even in those in whom prior anti-TNF therapy failed.

What are the new findings?

- This case shows that it can be a challenge to manage seronegative spondyloarthropathy in the context of pouchitis or active IBD.

- The combination of vedolizumab and anti-TNF-inhibitors might be a safe and effective treatment strategy.

- This case study may provide an impulse to investigate the efficacy and safety of the specific combination of TNF-inhibitors and integrin-inhibitors further, and to evaluate the combination of biological agents in general.

How might it impact on clinical practice in the foreseeable future?

- Etanercept may represent a useful adjunct to control active spondylarthropathy in patients with IBD showing only intestinal response to vedolizumab.

\section{CASE REPORT}

A 56-year-old man suffering from UC for 17 years was diagnosed with SpA 14 years ago. Symptoms of SpA developed parallel to manifestation of UC with lower back pain and morning stiffness.

The human leucocyte antigen B27-negative SpA presented with axial and peripheral involvement and initially showed clinical response on sulfasalazine and paracetamol. The 41-year-old patient underwent proctocolectomy with IPAA as a result of refractory pancolitis. Thereafter, he developed severe chronic refractory pouchitis. Subsequently various combinations of antibiotics, mesalazine, steroids and probiotics were 
administered with only transient response. Owing to increased joint pain and ongoing pouch inflammation treatment with a TNF-inhibitor adalimumab was initiated 3 years after surgery. While TNF inhibition led to control of SpA, activity of chronic pouchitis only partially improved. Despite several changes of treatment regime, especially switching to other TNF blocking agents, increase in dosage (consecutively infliximab, certolizumab pegol and golimumab) and combination with methotrexate no substantial clinical response was achieved. Owing to persistent pouchitis and a severe impact on his quality of life, inhibition of $\alpha 4 / \beta 7$-integrin was initiated with the therapeutic antibody vedolizumab (VDZ) at standard dose (300 $\mathrm{mg}$ at 0,2 and 6 weeks) and anti-TNF therapy was stopped. The application intervals were continued every 8 weeks. After 6 weeks of therapy with VDZ, the clinical symptoms of pouchitis disappeared, but SpA substantially worsened with clinical enthesiopathy, peripheral arthritis, significant vertebral pain, further stiffening, a rise in $\mathrm{C}$ reactive protein and a BASDAI-Index of 24. Therapy with TNF-inhibitor etanercept (ETA) (50 mg/week) was started in addition to VDZ.

After 20 weeks of VDZ endoscopic and histopathological assessment of the pouch revealed normal, uninflamed mucosa (figure 1). The additional application of ETA improved the joint symptoms after 1 week. After 4 weeks of therapy the BASDAI-Index decreased to 0 . After 10 months of treatment the patient is still without any symptoms of pouchitis or joint pain. No side effects of the combination of $\alpha 4 / \beta 7$-integrin and TNF-inhibition were seen.

\section{DISCUSSION}

This case is the report of a successful combination therapy of VDZ and ETA in patients with refractory pouchitis and additional seronegative SpA.

The precise aetiology of pouchitis is still unclear. Often pouchitis is because of a dysbalance of the microbial flora. Antibiotics are still the first-line therapy and patients with most acute pouchitis respond to antibiotic therapy. There are some retrospective studies, case series and case reports demonstrating the successful use of anti-TNF-agents (infliximab and adalimumab) in patients with refractory pouchitis. In a Spanish multicentre study, 31 patients with UC with chronic refractory pouchitis were treated with infliximab. After 8 weeks of treatment $21 \%$ of patients achieved complete response and $63 \%$ showed partial clinical response. Similar results have been reported in other studies. ${ }^{7}$ The response of pouchitis to immunomodulating therapies implies that not only dysbiosis, most likely also cytokines play a central role in the maintenance of pouchitis.

In our case VDZ was highly effective for the long lasting, refractory pouchitis. VDZ seems to be effective for maintaining remission as well. Especially for patients who suffer from chronic antibiotic refractory pouchitis and show a loss of response this seems to be an alternative therapeutic option. ${ }^{6}$ This presumption stands in line with results of other studies, which have shown that particularly patients with IBD who failed prior anti-TNF therapy showed good response to VDZ. ${ }^{8}{ }^{9}$ There is no data in the recent literature on the safety and efficacy of using VDZ in the management of refractory pouchitis.

VDZ was ineffective for SpA in our patient, whose symptoms even worsened under VDZ intervention. The additional application of ETA resulted in full control of SpA. Theoretically, a delayed benefit from VDZ could have improved the patient after 6 weeks when the ETA was administered. However, one has to point out that SpA symptoms were absent when the patient was started on VDZ because of his refractory pouchitis and the SpA relapsed after the switch from anti-TNF to VDZ.

Rubin et al performed a post hoc analysis of the Gemini II study to investigate the effect of VDZ on EIM. These data did not show a significant benefit of VDZ over placebo for the treatment of EIMs including arthritis and $\mathrm{SpA}$ in IBD. ${ }^{10}$ However, this retrospective analysis was limited by the fact that EIM of IBD was not assessed systematically on quantitative or semiquantitative scales in the Gemini studies.

In the Gemini studies, arthralgia was one of the most common adverse events affecting patients who received VDZ. ${ }^{9}{ }^{11}$ However, there was no significant difference of the incidences of arthralgia in comparison to the placebo group $(9.0-9.1 \%$ in Gemini II, $13.5-13.1 \%$ in Gemini II). This could suggest that VDZ itself does not cause arthralgia in terms of a side effect but rather seems to have no benefits on IBD-associated arthralgia with exacerbation of joint symptoms.

Our case demonstrates that the combination of VDZ and ETA might be an option to control both diseases. Anti-TNF is highly effective in the treatment of IBD as well as in SpA. However, up to one-third of the patients develop a primary and over one-third secondary loss of response. ${ }^{12}$ In our case there was a secondary loss of response to anti-TNF regarding IBD but maintaining response regarding SpA. Switching to VDZ was associated with exacerbation of joint inflammation. Although there are considerable overlaps in the treatment of IBD and SpA, biological therapies can result in differential efficacies. Therefore, the combination of an anti-Integrin and an anti-TNF could be a feasible treatment strategy to control both entities. In conclusion ETA can be used as an add-on therapy for patients with refractory IBD who showed response to vedolizumab concerning intestinal inflammation but suffering from SpA or other joint manifestation.

Based on the differential response to VDZ, it appears unlikely that SpA and IBD are pathophysiologically related to $\alpha 4 / \beta 7$ positive lymphocytes as previously suggested. $\alpha 4 / \beta 7$ Expressing lymphocytes are most likely not associated in the aetiology of SpA. In contrast, previous studies assumed that aberrant homing of mucosal $\mathrm{T}$ 
Figure 1 (A) Refractory pouchitis with a PDAl of $10,(B)$ pouch with normal mucosa and PDAI of 4 in week 20 after VDZ; (C) histological aspect of chronic refractory pouchitis, (D) histological aspect of uninflamed pouch mucosa after therapy. PDAI, Pouchitis Disease Activity Index; VDZ, vedolizumab.
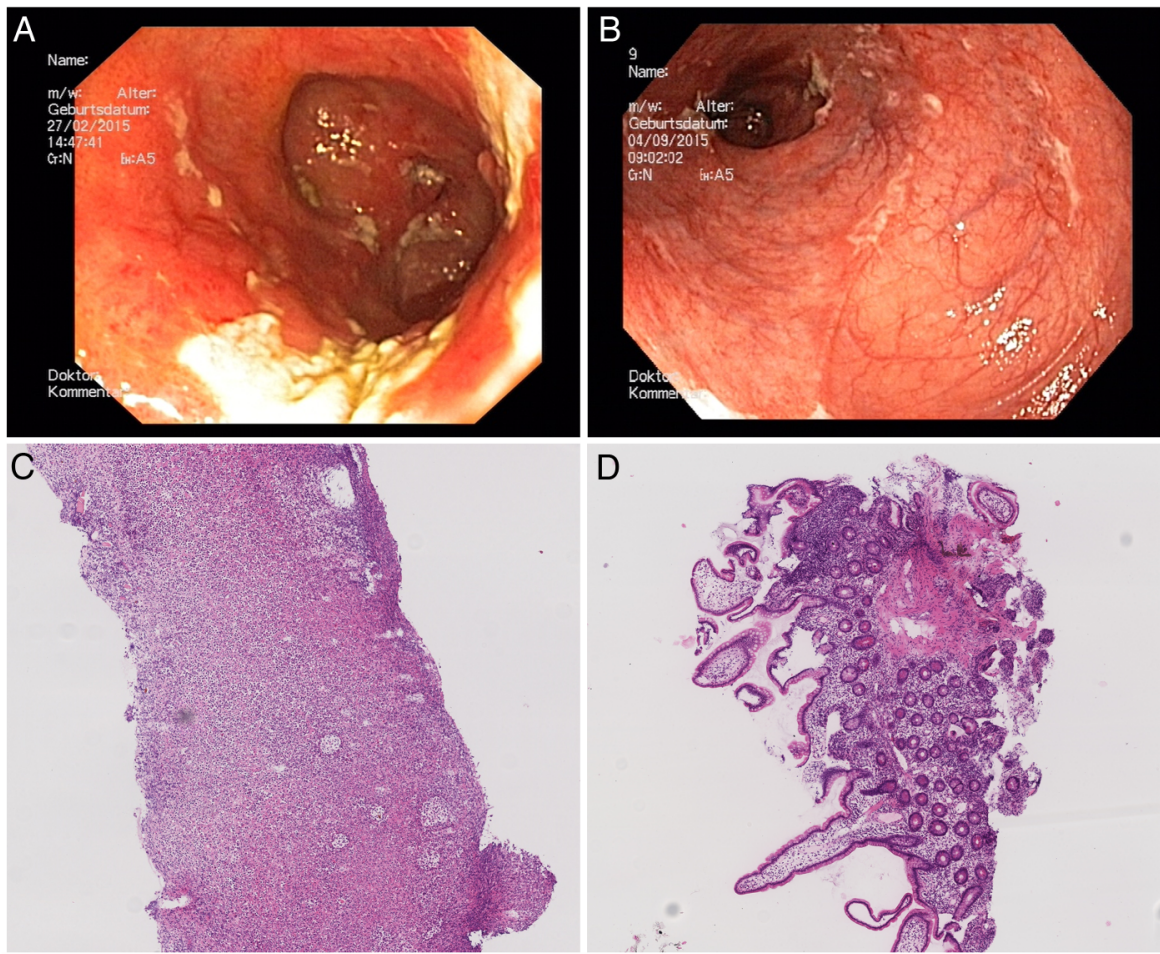

Open Access This is an Open Access article distributed in accordance with the Creative Commons Attribution Non Commercial (CC BY-NC 4.0) license, which permits others to distribute, remix, adapt, build upon this work noncommercially, and license their derivative works on different terms, provided the original work is properly cited and the use is non-commercial. See: http:// creativecommons.org/licenses/by-nc/4.0/

\section{REFERENCES}

1. Gionchetti $P$, Calabrese $C$, Rizzello F. Inflammatory bowel diseases and spondyloarthropathies. J Rheumatol Suppl 2015;93:21-3.

2. Brakenhoff LK, van der Heijde DM, Hommes DW. IBD and arthropathies: a practical approach to its diagnosis and management. Gut 2011;60:1426-35

3. Jacques $P$, Van Praet L, Carron $P$, et al. Pathophysiology and role of the gastrointestinal system in spondyloarthritides. Rheum Dis Clin North Am 2012;38:569-82.

4. Elewaut D, De Keyser F, Van Den Bosch F, et al. Enrichment of T cells carrying beta7 integrins in inflamed synovial tissue from patients with early spondyloarthropathy, compared to rheumatoid arthritis. J Rheumatol 1998;25:1932-7.

5. Singh S, Stroud AM, Holubar SD, et al. Treatment and prevention of pouchitis after ileal pouch-anal anastomosis for chronic ulcerative colitis. Cochrane Database Syst Rev 2015;(11):CD001176.

6. Herfarth $\mathrm{HH}$, Long MD, Isaacs KL. Use of biologics in pouchitis: a systematic review. J Clin Gastroenterol 2015;49:647-54.

7. Barreiro-de Acosta M, Garcia-Bosch O, Souto R, et al. Efficacy of infliximab rescue therapy in patients with chronic refractory pouchitis: a multicenter study. Inflamm Bowel Dis 2012;18:812-17.

8. Feagan BG, Rutgeerts $\mathrm{P}$, Sands BE, et al. Vedolizumab as induction and maintenance therapy for ulcerative colitis. $N$ Engl J Med 2013;369:699-710.

9. Sandborn WJ, Feagan BG, Rutgeerts $P$, et al. Vedolizumab as induction and maintenance therapy for Crohn's disease. $N$ Engl J Med 2013;369:711-21.

10. Rubin D, Feagan B, Dryden G, et al. The effect of vedolizumab on extraintestinal manifestations in patients with Crohn's disease in GEMINI 2. Inflamm Bowel Dis 2016;22:105.

11. Baumgart DC, Bokemeyer B, Drabik A, et al. Vedolizumab induction therapy for inflammatory bowel disease in clinical practice-a nationwide consecutive German cohort study. Aliment Pharmacol Ther 2016;43:1090-102.

12. Gisbert JP, Marin AC, McNicholl AG, et al. Systematic review with meta-analysis: the efficacy of a second anti-TNF in patients with 
inflammatory bowel disease whose previous anti-TNF treatment has failed. Aliment Pharmacol Ther 41:613-23.

13. Van Damme N, Elewaut D, Baeten D, et al. Gut mucosal T cell lines from ankylosing spondylitis patients are enriched with alphaEbeta7 integrin. Clin Exp Rheumatol 2001;19:681-7.

14. Alcazar Navarrete B, Quiles Ruiz-Rico N, Gonzalez Vargas F, et al. [Bronchiectasis following colectomy in a patient with ulcerative colitis and factor V Leiden mutation]. Arch Bronconeumol 2005;41:230-2.
15. Hirten R, Longman RS, Bosworth BP, et al. Vedolizumab and infliximab combination therapy in the treatment of Crohn's disease. Am J Gastroenterol 2015;110:1737-8.

16. Bonovas S, Fiorino G, Allocca M, et al. Biologic therapies and risk of infection and malignancy in patients with inflammatory bowel disease: a systematic review and network meta-analysis. Clin Gastroenterol Hepatol 2016;14: 1385-1397.e10. 